Methods Prior to the introduction of the PPS programme in July 2011 a Sedation Committee was established comprising a core group of senior nurses and a Consultant in Emergency Medicine. The committee developed the PPS programme including key educational elements (Sedation Manual; Lecture; treatment order form and checklist; Parent Information Leaflet) and credentialing through multiple-choice questions (online and open-book), bedside teaching ( 2 scheduled practice sessions) and 2 competency assessments (final clinical/moulage).

Results Since its inception (July 2011) a total of 48 ED staff members have started the PPS programme:

- 17 doctors (9 Registrars and 8 Senior House Officers) with 7 fully credentialled;

- 26 nursing staff (1 Clinical Nurse Manager (CNM) 3, 5 CNM2, 1 Advanced Nurse Practitioner (ANP) and 19 staff nurses) with 12 fully credentialled.

Conclusions The introduction phase of our ED PPS, the first of its kind in Ireland, has been successful. As a result of the multidisciplinary development process, the programme will likely have broad applicability in different types of ED, and potentially other clinical areas, caring for children.

\section{PHARMACISTS INTERVENTIONS IN A CHILDRENS HOSPITAL - WHAT CAN THEY TELL US? DO THEY PREVENT HARM?}

doi:10.1136/archdischild-2012-302724.1558

RE Isaac, A Hussain. Pharmacy Department, Birmingham Children's Hospital NHS Foundation Trust, Birmingham, UK

Background Clinical Pharmacists review prescriptions for safe and economic use. Prescribing for children involves increased complexity and errors have potentially more serious consequences.

Method Paediatric pharmacists' interventions logs were entered into a database and analysed. A harm category using the National Coordinating Council for Medication Error Reporting and Prevention (MERP) algorithm (1) and type of intervention was assigned to each. Some interventions records were assigned more than one type e.g. renal impairment and wrong frequency.

Results Of the 500 records, 489 interventions prevented harm as seen in the table below (category A-D).

\section{Abstract 1558 Table 1 Table of MERP Categories}

\begin{tabular}{cccccc}
\hline A & B & C & D & E & G \\
53 & 163 & 145 & 128 & 10 & 1 \\
\hline
\end{tabular}

Interventions were not captured before administration on 260 $(52 \%)$ occasions, 113 of thee were via the parenteral route. Wrong dose was cause for intervention in $41.8 \%$ prescriptions (115 dose too high, 94 underdose) and incorrect frequency in 67 (13.4\%). There were 18 interventions involving wrong calculation, decimal point or unit of mass errors. Formulation issues were the cause of $38(7.6 \%)$ interventions which $7 / 38$ also involved cost savings. Altered drug handling e.g renal impairment, prematurity was involved in $105(21 \%)$ interventions. Ambiguous prescribing or legalities led to $58(11.6 \%)$ interventions. There were $35(7 \%)$ unintentional errors as a result of incorrect or incomplete drug history taking on admission.

Conclusions Paediatric prescribing errors reaching and harming paediatric patients can be reduced as a result of timely intervention by pharmacists.

\section{Reference:}

1. http://www.nccmerp.org/pdf/algorColor2001-06-12.pdf. 1559 BUTTON BATTERY INJURIES LODGED IN THE ESOPHAGUS,
THE EVOLVING DANGER

doi:10.1136/archdischild-2012-302724.1559

M Cevik, ME Boleken. Pediatric Surgery, Harran University, Medical Faculty, Sanliurfa, Turkey

Background and Aim Button battery (BB) ingestion is potentially a life-threatening condition for children and has increased continuously in recent years. BBs lodged in the esophagus may cause serious complications and even death. The aim of the present study was to compare the relevant studies in the literature to our results of cases in which a BB was lodged in the esophagus.

Methods The present study retrospectively analyzed 16 patients who ingested BBs that lodged in the esophagus. Data were collected from medical charts at the pediatric surgery department during 2007-2011. Ten male (62.5\%) and six female patients aged 2-99 months (mean age $\pm \mathrm{SD}, 34.81 \pm 25.23$ months) were evaluated. Cases were studied for time and location of the battery in the esophagus, presenting symptoms, diagnostic evaluation, complications, and outcomes.

Results Children who ingested BBs were all $<6$ years of age (14) (87.4\%). The most common clinical complaint of the patients was history of swallowing and diysphagia. Eight patients suffered from corrosion at different stages, 2 had an eosophageal perforation and tracheoesophageal fistula. Two patients were death releated complication of BB ingestion. Nine (56.3\%) patients had BB ingestion history before admission to the clinic. All $\mathrm{BBs}$ were from toys and were lithium $\mathrm{BBs}$ of $>15 \mathrm{~mm}$ circumference.

Conclusions $\mathrm{BB}$ ingestion is an important condition in children. An endoscopic examination and removal must be performed urgently for a BB lodged in the esophagus.

\section{PARENTAL SUPERVISION MAY NOT BE ENOUGH IN PREVENTING ACCIDENTAL POISON INGESTION IN CHILDREN}

doi:10.1136/archdischild-2012-302724.1560

S Bharwani. Pediatrics, Faculty of Medicine and Health Sciences, UAE University, Al Ain, United Arab Emirates

Background/Aims It is a general notion that accidental poison ingestions in children seldom happen under parental supervision. Our objective was to compare the doctors' perceptions of supervision with the parental recall of the actual events.

Methods Pediatricians were recruited for a short survey and asked using Likert scale if they agree/disagree that children are more likely to ingest harmful substances when supervised by their parents. Parents of the children who had been seen in the ER (emergency room) in the preceding 3-13 months with accidental poison ingestion were called and asked who first discovered the child ingest a harmful substance. The answers were compared using frequency bar plotting to get the percentage of responses and referenced against the documented medical records.

Results 107 doctors out of 136 approached responded to this question item. 71 mothers were willing to talk about their experience from the 100 parents. 13 physicians out of 107 (12\%) believed that children were more likely to ingest harmful substance when they are supervised by their parents. From the 71 parents interviewed 51(72\%) recalled that either the mother or the father or both were present when the child ingested a harmful substance. From the hospital record 86 out of 101 cases (85\%) documented parent/s as witnesses. The difference between the physicians' perceptions and the parental record plus the hospital data was significant ( $\mathrm{p}=0.000$, CI 95\% 1.75-2.09).

Conclusion Parental supervision is not a $24 / 7$ surveillance and in itself not an adequate strategy in preventing accidental poison ingestions. 